\title{
Grupo de pais: aprendizagens de participantes do Programa ACT
}

\author{
Parent Group: ACT Program participants new learnings
}

\section{Grupo de padres: aprendizajes de los participantes del programa ACT}

\author{
Joyce Lúcia Abreu Pereira Oliveira1 ORCID 0000-0001-7038-5856 \\ Milena Carolina Fiorini ${ }^{2}$ ORCID 0000-0003-1390-1564 \\ Luciane Guisso ${ }^{3}$ ORCID 0000-0002-4846-319X \\ Mauro Luís Vieira ${ }^{4}$ ORCID 0000-0003-0541-4133 \\ Maria Aparecida Crepaldi ${ }^{5}$ ORCID 0000-0000-0002-5892-7330
}

\section{Universidade Federal de Santa Catarina. Brasil.}

\begin{abstract}
Resumo: O objetivo deste estudo foi identificar as aprendizagens de participantes de grupos de pais do Programa ACT, intervenção psicoeducativa que visa auxiliar no desenvolvimento de práticas educativas parentais positivas, propiciando às crianças ambientes mais seguros, saudáveis, estáveis, sem violência e maus-tratos. Os dados foram organizados de acordo com a análise categorial temática. Os resultados indicaram que a participação no ACT possibilitou aprendizagens sobre práticas educativas parentais, o reconhecimento de emoções dos adultos e das crianças, a compreensão acerca dos estilos parentais, a importância do envolvimento dos pais para o desenvolvimento da criança, além de aprendizagens sobre desenvolvimento infantil e aspectos relacionados a exposição às mídias eletrônicas na primeira infância. Este estudo apresentou como contribuição às pesquisas do Programa ACT uma análise qualitativa de aspectos específicos concernentes às aprendizagens realizadas nas sessões e resultados de grupos que incluíram a participação de pais (homens) no contexto brasileiro, diferenciando-se de estudos anteriores apenas com mães.
\end{abstract}

Palavras-chave: relações pais-filhos, treinamento de pais, grupo de pais, parentalidade, Programa ACT.

Abstract: The aim of this study was to identify the new learning of participants of an ACT Program parent group. This psychoeducational intervention aims to assist in the development of positive parenting practices, providing children safer, healthier, more stable environments, without violence or abuse. Data were organized according to thematic categorical analysis. The results indicated that participation in the ACT Program enabled learning about parenting practices, the recognition of emotions of the adults and children, understanding about parenting styles, the importance of parental involvement for child development, and learning about child development and aspects related to exposure to electronic media in early childhood. As a contribution to the ACT Program research, this study presented a qualitative analysis of specific aspects concerning the content learned in the sessions and results of groups that included the participation of fathers in the Brazilian context, differing from previous studies with only mothers.

Keywords: parent-child relations, parent training, parent group, parenting, ACT Program.

(cc) $E Y$

This work is under a Creative Commons Atribution 4.0 International License 
Resumen: El objetivo de este estudio fue identificar los aprendizajes de los participantes de los grupos de padres del Programa ACT, intervención psicoeducativa que tiene como objetivo ayudar en el desarrollo de prácticas parentales positivas, proporcionando a los niños entornos más seguros, saludables y estables, sin violencia y abuso. Los datos se organizaron según el análisis categórico temático. Los resultados indicaron que la participación en el Programa ACT permitió aprender sobre prácticas educativas, el reconocimiento de las emociones de adultos y niños, la comprensión de estilos parentales, la importancia de la participación de los padres para el desarrollo infantil, así como el aprendizaje sobre el desarrollo infantil y los aspectos relacionados a la exposición a los medios electrónicos em la primera infancia. Este estudio presentó como contribución a la investigación del Programa ACT un análisis cualitativo de aspectos específicos del aprendizaje realizado en las sesiones y resultados de grupos que incluyeron la participación de padres (hombres) en el contexto brasileño, diferenciándose de estudios previos solo con madres.

Palabras clave: relaciones padres-hijos, formación de padres, grupo de padres, crianza, Programa ACT.

Como citar:

Oliveira, J. L. A. P., Fiorini, M. C., Guisso, L., Vieira, M. L., \& Crepaldi, M. A. (2021). Grupo de pais: aprendizagens de participantes do Programa ACT. Ciencias Psicológicas, 15(1), e-2392. doi: https://doi.org/10.22235/cp.v15i1.2392

Correspondência: Laboratório de Psicologia da Saúde, Família e Comunidade - Universidade Federal de Santa Catarina. Centro de Filosofia e Ciências Humanas. Departamento de Psicologia. Campus Reitor João David Ferreira Lima, s/n, Florianópolis-SC, Brazil. CEP 88.040-970. E-mail: joycelapo@gmail.com

Os pais ${ }^{1}$ desempenham um papel crucial para o desenvolvimento da criança, pois são os principais responsáveis por criar condições básicas de sobrevivência, proteção, socialização e afeto (Dessen \& Polonia, 2007). Em um contexto ideal, eles deveriam atuar como os principais provedores de mecanismos de proteção, satisfazendo as necessidades da criança em termos de carinho, cuidados e estabilidade, fatores que compõem uma parentalidade positiva (Linhares, 2015; Pereira, Goes, \& Barros, 2015). As interações entre pais e filhos, caracterizadas por envolvimento parental, vêm sendo amplamente estudados, especialmente a relação pai-filho, tendo em vista a modificação da participação deste nas últimas décadas em relação ao cuidado infantil (Barbeta \& Cano, 2017; Lamb, Pleck, Charnov, \& Levine, 1985; Schmidt et al., 2019).

Como forma de promover práticas educativas parentais positivas, a Organização Mundial da Saúde - OMS aponta a importância da participação de pais em programas de intervenção que busquem ampliar o conhecimento acerca do desenvolvimento infantil e incentivar relacionamentos familiares saudáveis (Pereira et al., 2015; Weymouth \& Howe, 2011). Uma revisão de literatura realizada por Altafim e Linhares (2016) identificou 16 diferentes programas universais de treinamento parental. Três desses programas apresentam

\footnotetext{
${ }^{1} \mathrm{O}$ termo "pais", neste estudo, abarca os progenitores de ambos os sexos (biológicos ou adotivos) e o termo "pai", no singular, refere-se exclusivamente ao progenitor do sexo masculino.
} 
maiores evidências de efetividade (Linhares, 2015) e são reconhecidos pela Organização Mundial da Saúde - OMS: Programa Triple P (Sanders, 2008), Programa Incredible Years (Webster-Stratton, Reid \& Hammond, 2001) e Programa ACT - Raising Safe Kids Parenting Program (Silva, 2011). Os participantes dos programas de intervenção citados são os responsáveis legais pelos cuidados da criança, uma vez que investir em interações seguras e positivas entre pais e filhos, constitui-se na base para o desenvolvimento infantil saudável (Sanders, Kirby, Tellegen, \& Day, 2014).

O Programa ACT, foco deste estudo, foi desenvolvido nos Estados Unidos em 2001, pela Divisão de Prevenção da Violência da American Psychological Association e destinase a pais de crianças com idades entre zero a oito anos (Silva, 2011). O principal objetivo do Programa ACT é auxiliar os pais no desenvolvimento de práticas educativas parentais positivas, propiciando às crianças ambientes mais seguros, saudáveis, estáveis, sem violência e maus-tratos (Knox, Burkhart, \& Howe, 2011; Silva \& Willians, 2016). O programa busca melhorar as práticas educativas parentais por meio da transmissão de conhecimentos sobre o desenvolvimento infantil, ensino de estratégias de regulação emocional e disciplina positiva, além de incentivara participação nos esforços da comunidade relativos à prevenção da violência (Altafim, 2017; Silva, 2011).

Embora a implantação do Programa ACT esteja em expansão mundial, ainda existem poucas pesquisas científicas relacionadas à intervenção. Dentre os estudos internacionais, os resultados de pesquisas quantitativas têm demonstrado, principalmente, a efetividade do Programa ACT para a redução de práticas educativas parentais negativas (Knox \& Burkhart, 2014; Knox, Burkhart, \& Cromly, 2013; Knox, Burkhart, \& Hunter, 2010; Portwood, Lambert, Abrams, \& Nelson 2011; Weymouth \& Howe, 2011). Os achados dos estudos quantitativos brasileiros envolvendo o Programa ACT apontam para a ampliação das práticas educativas positivas maternas (Altafim, 2017; Altafim, Pedro, \& Linhares, 2016; Camargo, 2016; Pedro, 2016; Pontes, 2015; Silva \& Williams, 2016), redução do uso de práticas negativas abusivas e/ou violentas das mães e cuidadoras (Altafim, 2017; Altafim et al., 2016; Pedro, 2016; Silva \& Williams, 2016), e diminuição de problemas de comportamento das crianças (Altafim, 2017; Pedro, 2016).

Percebe-se que há consenso entre os achados de pesquisas desenvolvidas até o momento, sobretudo no que diz respeito ao impacto positivo do Programa ACT nas práticas educativas parentais. Porém, assim como no exterior, no cenário brasileiro ainda há resultados limitados no que se refere à avaliação qualitativa do Programa ACT.A investigação de Pontes (2015), que utilizou procedimentos observacionais da interação paiscriança como parte da investigação, não encontrou diferenças significativas na frequência observada de comportamentos positivos ou negativos entre pais e filhos antes e após a aplicação do Programa ACT. No entanto, a autora observou aumento do conhecimento dos pais acerca do desenvolvimento infantil e do comportamento parental.

Ademais, as pesquisas no Brasil têm priorizado grupos de mães (Altafim, 2017; Altafim et al., 2016; Pedro, 2016; Silva \& Williams, 2016) e sabe-se até o momento de apenas um estudo que teve um pai como participante do programa (Pontes, 2015). Desse modo, estudos que incluem pais (homens) e cuidadores mostram-se relevantes no cenário brasileiro.

Nesse sentido, além dos dados quantitativos obtidos pelas investigações, resultados que demonstrem qualitativamente a percepção dos participantes brasileiros em relação às aprendizagens deles com o Programa ACT representam fontes de informação pertinentes para avaliar se as finalidades do programa estão sendo atendidas em âmbito nacional. 
Ademais, alerta-se para a importância de compreender de que forma a participação dos pais no Programa ACT influencia as suas estratégias de cuidado e educação dos filhos.

Diante desse cenário, o objetivo do presente estudo foi identificar as aprendizagens realizadas por participantes de grupos do Programa ACT, com base em uma das atividades realizadas ao longo das sessões do programa. O emprego do termo 'aprendizagem', nesta pesquisa, foi fundamentado na Teoria da Aprendizagem Social de Bandura (1977). De acordo com o autor, as habilidades são aprendidas por meio de observação e experiência direta, e mantidas ou alteradas pelas suas consequências sociais. $O$ processo de desenvolvimento das aprendizagens decorre, portanto, da inter-relação entre estímulos externos (fatores sociais e ambientais) e internos (processos cognitivos). No contexto do Programa ACT, espera-se que os pais aprendam novas habilidades por meio da observação de conteúdos e de comportamentos de outros participantes, bem como da aplicação dessas habilidades no âmbito familiar. Como o ACT vêm sendo aplicado em contextos socioeconômicos e culturais distintos, identificar as aprendizagens evidenciadas pelos participantes é uma forma de aprimorar a intervenção, a fim de que possíveis ajustes/melhorias possam ser considerados na realidade brasileira, além de embasar políticas públicas direcionadas a famílias com crianças pequenas.

\section{Método}

\section{Participantes}

Foram realizados seis grupos de intervenção do Programa ACT, sendo quatro em instituições públicas (creches e escolas) e dois em uma empresa privada, no período de outubro de 2017 a dezembro de 2018, em uma cidade do Sul do Brasil. O total de iniciantes foi de 69, porém 45 pessoas concluíram. Neste estudo, foram incluídos somente os pais que participaram de, no mínimo, seis sessões do Programa ACT, além da reunião prévia, totalizando 32 participantes (27 mulheres e cinco homens).

\section{Procedimentos}

Esta pesquisa qualitativa faz parte de um projeto mais amplo denominado "ACT Programa de treinamento parental para pais de crianças de zero a oito anos de idade", aprovado pelo Comitê de Ética em Pesquisa com Seres Humanos da Universidade Federal de Santa Catarina, sob o $\mathrm{n}^{\circ}$ de parecer 2.766.095. Os procedimentos seguiram os parâmetros éticos sobre a Pesquisa com Seres Humanos nas Ciências Humanas e Sociais, da Resolução CNS 510/16 (Conselho Nacional de Saúde, 2016). A participação dos pais foi voluntária, respaldada pela garantia de seu anonimato e pela assinatura do Termo de Consentimento Livre e Esclarecido - TCLE. Os grupos de intervenção foram facilitados por três psicólogas, doutorandas de um Programa de Pós-Graduação em Psicologia, devidamente certificadas como facilitadoras do Programa ACT, com auxílio de estudantes de graduação em Psicologia, treinadas como co-facilitadoras.

A intervenção do Programa ACT é estruturada em uma reunião prévia, seguida de oito sessões semanais. Cada encontro tem duração de duas horas cada e apresenta temáticas pré-definidas. O grupo de intervenção deve conter entre seis e doze pais participantes (Silva, 2011). Os encontros são realizados na seguinte sequência: $1^{\circ}$ encontro: Reunião prévia do Programa; sessão 1: compreensão do comportamento e desenvolvimento infantil; sessão 2: a violência na vida das crianças; sessão 3: manejo da raiva no adulto; sessão 4: como ajudar a criança na regulação emocional, incluindo o controle da raiva; sessão 5: a influência dos 
meios eletrônicos no comportamento infantil; sessão 6: disciplina e estilos parentais; sessão 7: disciplina voltada a comportamentos positivos; e sessão 8: a função dos pais, no sentido de promover ambientes seguros para as crianças. Os encontros do Programa ACT são interativos, articulando exposição de conteúdo, reflexões, discussões, dinâmicas em grupo e tarefas.

O presente estudo apresenta os resultados da análise de uma das atividades do programa, realizada entre as sessões 1 e 7 do programa. Essa atividade consiste no seguinte processo: no término de cada encontro, os participantes receberam um pequeno folheto com a pergunta: "Quais são as duas coisas/ferramentas que você aprendeu hoje e que serão úteis para alcançar seus objetivos para seus filhos?" (Silva, 2011). Os pais tinham, então, alguns minutos para responder e guardavam o folheto preenchido em uma caixa individual. Todas essas respostas dos participantes foram transcritas e somaram 342 frases, resultantes dos seis grupos realizados.

\section{Análise dos dados}

Os dados foram organizados com auxílio do Software ATLAS.ti versão 8.0 e analisados de acordo com a análise categorial temática de Bardin (2011), que inclui três fases: 1. Pré-análise; 2. Exploração do material; e 3. Tratamento dos resultados, inferência e interpretação. Na primeira etapa (pré-análise) realizou-se a leitura minuciosa dos dados, a definição do corpo de análise. Na segunda etapa (exploração do material), os dados foram classificados em 457 unidades de registro, a partir de recortes das frases dos participantes, incluindo pais e mães, o que resultou em 16 subcategorias, sintetizadas em 6 categorias. $\mathrm{Na}$ terceira fase foi realizado o tratamento dos resultados, inferência e interpretação.

O sistema de categorias construído foi submetido à análise de duas juízas, pesquisadoras e terapeutas de família. O índice de concordância Inter juízes foi de $87 \%$ e a partir desse procedimento alguns ajustes foram realizados no sistema de categorias.

\section{Resultados}

Os resultados foram organizados em seis categorias, conforme apresentado a seguir, juntamente às respectivas frequências: i) Aprendizagem de práticas educativas parentais (160); ii) Reconhecimento e controle de emoções dos pais e da criança (100); iii) Envolvimento parental (70); iv) Compreensão acerca dos estilos parentais (47); v) Controle da utilização de mídias eletrônicas (43); e vi) Compreensão sobre o desenvolvimento da criança (37).

\section{Aprendizagem de práticas educativas parentais}

Esta categoria refere-se às estratégias de disciplina, orientação, cuidado e socialização aprendidas pelos participantes durante a intervenção parental. Foram recorrentes os apontamentos dos pais sobre a importância da disciplina positiva, evidenciando a necessidade de colocar regras e limites claros às crianças, sem a utilização de estratégias punitivas. Os pais escreveram, ainda, expressões que representam a valorização de comportamentos positivos dos filhos, com ênfase em elogios e recompensas, como, por exemplo, "aprendi a valorizar as qualidades e não focar só nos defeitos". A relevância da construção de uma relação afetiva com os filhos, com respeito, amor, carinho, gentileza, empatia e cuidado também foi evidenciada em frases, como "ter carinho e dar bastante amor"; e "mais respeito com as crianças, dar mais carinho a elas". 
O reconhecimento dos efeitos deletérios das práticas educativas parentais negativas também foi observado através de registros, como, "evitar agressões fisicas e verbais"; "não bater"; "não gritar"; "não fazer o filho se sentir mal quando não se comporta bem" e a compreensão das consequências negativas dessas práticas ao longo de todo o ciclo de vida da criança também foi relatada por um participante, "violência de todos os tipos causam marcas e cicatrizes que não se apagam".

Além disso, as anotações dos pais evidenciaram a desnaturalização da violência, ou seja, a percepção de que a negligência e a violência psicológica são tão prejudiciais quanto a violência física, a exemplo das seguintes frases "eu posso evitar diversos tipos de exposição à violência" e "violência não é só fisica". A importância de ajudarem os filhos na resolução de conflitos também estava presente nos apontamentos dos participantes, "devo propor soluções para meus filhos lidarem com conflitos", "ajudar os filhos a solucionar conflitos". Os participantes salientaram a importância de identificar a causa dos conflitos com os filhos e/ou entre os filhos, bem como as emoções envolvidas na situação e construir alternativas de soluções em conjunto com a criança.

Os pais apontaram a importância do diálogo entre os pais e a criança, a exemplo da frase "saber escutar meu filho para poder ajudá-lo". Além disso, os participantes colocaram o diálogo como uma ferramenta fundamental em diferentes situações com a criança, expressando que "o diálogo sempre é a melhor opção" e "para ensinar a dialogar, eu preciso dialogar”.

\section{Reconhecimento e manejo de emoções dos pais e da criança}

Nesta categoria, foram incluídos registros relacionados ao reconhecimento da importância das emoções e dos sentimentos na relação com a criança, no sentido de identificação, controle e expressão, tanto do adulto quanto da criança. As anotações dos participantes, destacaram os termos "acalmar-se" e "paciência", os quais parecem demonstrar o entendimento sobre a importância da regulação emocional dos pais no manejo com os filhos: "aprendi a ter mais calma e paciência, pensar antes de reagir". Outro aspecto notável nos registros foi a consideração das aprendizagens para lidar com as próprias emoções: "controlar a raiva (meu estado de raiva)"; "lidar melhor com a raiva, adotar estratégias para conseguir lidar com ela".

Foi evidenciado nos registros a aceitação e naturalização da raiva como uma emoção que faz parte da natureza humana, a qual pode ser identificada através de sinais físicos (aceleração dos batimentos cardíacos, suor, etc.), cognitivos (pensamentos) e comportamentais: "estar atenta aos sinais que o corpo dá antes da gente explodir"; "sentir como meu corpo reage quando estou com raiva"; "ajudar a criança a compreender melhor suas emoções através de conversa, após me acalmar". O entendimento sobre a importância da expressão de emoções como a raiva, de modo saudável, também foi recorrente entre os registros: "aprendi a lidar com os sentimentos de raiva, principalmente a compreender os sentimentos, escutar um ao outro".

As notas destacaram, também, o reconhecimento da importância do adulto no sentido de auxiliar a criança a usar as palavras para expressar o que sente de modo assertivo, sem agressão ou violência. Evidenciaram também o quanto os pais são co-reguladores emocionais da criança e o quanto estão implicados no processo de aprendizagem emocional: "a raiva é um sentimento normal e devemos ensinar os filhos a lidar com esse sentimento e aprender sobre ele". 


\section{Envolvimento parental}

Esta categoria integra os registros sobre o suporte e o engajamento dos pais na realização de tarefas, atividades e brincadeiras que contribuem para o desenvolvimento psicossocial da criança. Nas aprendizagens descritas pelos pais observou-se $o$ reconhecimento da importância de que dediquem tempo de qualidade aos filhos, compreendam as diferentes fases do desenvolvimento infantil e qualifiquem a participação da figura paterna. A compreensão a respeito da relevância do envolvimento do pai no cuidado dos filhos pode ser observada na seguinte frase, escrita por um progenitor do sexo masculino "importância de observar e compartilhar, ou seja, antes de deixar a filha à deriva nas informações e conflitos, experimentar, refletir e depois expor; além de curtir com ela".

$\mathrm{O}$ engajamento parental em tarefas e atividades com os filhos, bem como o compartilhamento de vivências e brincadeiras apareceram nos apontamentos dos participantes, quando indicaram, "ajudar nos estudos", "ensinar a compartilhar", "importância de monitorar e assistir juntos filmes, desenhos e jogos com as crianças, e falar a respeito". Além disso, os pais apontaram a relevância da brincadeira no processo de aprendizado da criança: "aprendi a brincar com eles e a respeitar".

\section{Compreensão acerca dos estilos parentais}

Esta categoria agrega os registros dos pais acerca dos diferentes estilos parentais empregados no cuidado e na educação da criança, bem como dos fatores que influenciam a adoção desses estilos. Os participantes mostraram compreender que seu comportamento frente a diferentes situações e na relação com os filhos é uma referência para a criança, pois ela tende a reproduzir os mesmos comportamentos. As frases "nós somos os espelhos dos nossos filhos" e "as crianças tendem a reproduzir o que viveram" ressaltam a aprendizagem dos pais acerca do fato de que as crianças aprendem por meio de modelos, imitando os comportamentos dos adultos.

Os participantes também mostraram compreender que podem escolher pelo melhor estilo parental, através de suas percepções acerca da importância do equilíbrio entre autoritarismo e permissividade na educação dos filhos. Os participantes destacaram a vontade de se tornarem pais com autoridade, a exemplo dos seguintes apontamentos: "usar da autoridade sem ser autoritário" e "me tornar uma mãe no estilo do comportamento de pais com autoridade". Os pais evidenciaram, ainda, o reconhecimento de que seu estilo parental sofreu influência da forma como eles mesmos foram educados. Nesse sentido, assinalaram a pertinência de criar consciência a respeito dos estilos parentais dos próprios pais, buscando mudar aspectos negativos e enfatizar fatores positivos. A frase "refletindo sobre os meus traumas eu posso poupá-lo de muitos erros" retrata essa compreensão.

\section{Controle da utilização de mídias eletrônicas}

Nesta categoria, foram agregadas as anotações dos pais relacionadas ao monitoramento do conteúdo e tempo de exposição da criança às mídias eletrônicas (televisão, celular, tablets e videogames), bem como à utilização positiva dessas mídias na educação dos filhos. Os participantes mostraram compreender a relevância do monitoramento, tanto do tempo de acesso às mídias eletrônicas pela criança, quanto do conteúdo acessado por ela. A frase "devemos reduzir o tempo que ele fica exposto à TV e ter cuidado com o que ele está assistindo”, por exemplo, ressalta a preocupação dos pais com o tempo de exposição dos filhos aos meios eletrônicos e o conteúdo que estes assistem. Destacaram-se, também, apontamentos como "proporcionar momentos de brincar sem o 
uso de mídias", que denotam a necessidade de substituir as mídias eletrônicas por outras atividades no cotidiano da criança.

A utilização positiva das mídias eletrônicas também foi destacada, através de conversas com a criança sobre o conteúdo das mídias e a diferenciação entre fantasia e realidade. $\mathrm{O}$ registro "mostrar aos filhos que existe fantasia e realidade, que ele tem que brincar mais e deixar o mundo eletrônico de lado" expressa a aprendizagem dos participantes no sentido de estabelecer uma mediação diante do que a criança acessa, explicando que determinadas situações observadas na televisão, no celular ou em videogames não representam a realidade. Por fim, o apontamento "importância de monitorar e assistir juntos filmes, desenhos e jogos com as crianças, e falar a respeito" é um exemplo da percepção dos pais de que as mídias eletrônicas, se bem utilizadas, podem se configurar como um momento de troca de experiências com os filhos, especialmente se envolverem conteúdos educativos.

\section{Compreensão sobre o desenvolvimento da criança}

Nesta categoria, foram reunidos os registros acerca da compreensão do processo de desenvolvimento infantil por parte dos pais, incluindo os fatores que caracterizam o comportamento e o processo emocional da criança em diferentes faixas etárias, além das particularidades que envolvem temperamento e aspectos contextuais. Observou-se nos registros dos participantes a compreensão dos pais de que a criança passa por diversas fases de desenvolvimento psicossocial e cognitivo, de acordo com a idade, de modo que cada uma envolve comportamentos e estratégias de cuidado e educação específicas por parte dos pais. Os aspectos citados puderam ser observados nos seguintes registros: "pesquisar/entender a etapa de desenvolvimento da criança", e "entender que cada idade passa por uma fase/comportamento". Verificou-se, também, a importância do compartilhamento de informações entre pais com filhos em diferentes faixas etárias, conforme registrado: "o fato de estar aprendendo sobre a teoria e os exemplos das colegas sobre as fases que estão por vir auxiliam a me preparar para como estabelecer limites saudáveis desde já”. E, por fim, a percepção de que a forma como a criança lida e expressa as emoções tende a mudar ao longo do seu desenvolvimento, a exemplo das anotações "como a raiva se manifesta em diferentes idades" e "as crianças manifestam a raiva de diferentes formas, de acordo com a idade".

Por fim, a análise dos dados mostrou que os participantes compreenderam que certos comportamentos da criança são esperados, e fazem parte do processo de desenvolvimento, variando de acordo com o temperamento e outras questões contextuais, não somente a idade. Os pais indicaram essa aprendizagem nas seguintes frases: "cada criança tem seu tempo e se desenvolve de maneiras diferentes, devemos respeitar cada um" e "preciso respeitar os limites dos meus filhos, pois cada um tem um desenvolvimento particular". Assinalaram o cuidado com as expectativas depositadas nos filhos, conforme expresso: "analisar sempre: quais as expectativas estou colocando no meu filho (e, eventualmente, me frustrando)". Indicaram, também, a compreensão de que "a birra é uma revolta da criança geralmente por uma expectativa não atendida" e que é fundamental "entender a linguagem da criança: o que ela quer sinalizar com seu comportamento".

\section{Discussão}

Este estudo buscou identificar as aprendizagens realizadas por participantes de grupos do Programa ACT. De modo geral, os registros dos participantes mostraram-se 
condizentes com os principais objetivos do programa, que se referem à disseminação de conhecimentos a respeito do processo de desenvolvimento da criança, da influência das práticas educativas parentais e do estilo parental no desenvolvimento dos filhos, da regulação emocional tanto dos pais quanto da criança e do impacto dos meios eletrônicos na vida dos filhos (Altafim, 2017; Silva, 2011). Ao observar a denominação das categorias e subcategorias resultantes, destaca-se que os registros dos participantes retrataram fielmente os conteúdos trabalhados ao longo da intervenção.

Em conformidade com achados de pesquisas quantitativas realizadas no contexto do Programa ACT, observou-se a percepção dos pais acerca da necessidade de melhoria nas práticas educativas parentais (Altafim, 2017; Knox \& Burkhart, 2014; Knox et al., 2010; Knox et al., 2013; Pedro, 2016; Portwood et al., 2011; Weymouth \& Howe, 2011). Também em consonância com estudos anteriores, foi possível identificar a compreensão acerca da importância da utilização de práticas educativas positivas por parte dos pais, envolvendo aumento do comportamento afetuoso (Knox \& Burkhart, 2014; Knox et al., 2013; Portwood et al., 2011), além da aprendizagem da disciplina positiva (Altafim, 2017; Altafim, McCoy, \& Linhares, 2018). O reconhecimento dos participantes dos efeitos negativos de práticas educativas negativas também já foi evidenciado por outras investigações com o Programa ACT, que demonstraram, inclusive, a redução da violência psicológica e física na educação dos filhos (Miguel \& Howe, 2006; Knox et al., 2010, 2013; Porter \& Howe, 2008; Portwood et al., 2011). Nesse estudo, apesar da análise dos dados mostrar que os pais assimilaram esse entendimento, não se pode afirmar que reduziram as práticas negativas com os filhos.

No que concerne à aprendizagem de estratégias de análise e resolução de conflitos com a criança, os resultados são coerentes com a pesquisa internacional de Gulliford, Deans, Frydenberg e Liang (2015) e nacional de Pontes (2015), que verificaram aumento significativo na habilidade de resolução de problemas com os filhos após a participação dos pais no Programa ACT. Outro importante aspecto destacado pelos participantes foi a importância do diálogo com as crianças, em concordância com os dados revelados por Gulliford et al. (2015) e Altafim et al. (2018), sobre a melhoria na comunicação familiar entre participantes do Programa ACT

Quanto ao reconhecimento e manejo de emoções, a análise dos dados sugere que os participantes ampliaram a compreensão sobre as emoções, sobretudo, entenderam que a raiva é uma emoção natural, sentida por todos os indivíduos e que ao ser reconhecida desse modo, podem desenvolver aprendizagens sobre gestão emocional. Os dados sugerem que os pais compreenderam que as habilidades de regulação emocional podem ser desenvolvidas e com isso podem tornar-se bons co-reguladores emocionais dos filhos. Há evidências de que a consciência emocional relaciona-se à regulação efetiva das emoções (Boden \& Thompson, 2015) e pesquisas anteriores com o Programa ACT destacaram melhorias na regulação emocional (Altafim et al., 2018; Weymouth \& Howe, 2011) e comportamental (Altafim et al., 2018) dos participantes.

Em relação ao envolvimento parental, verificou-se a percepção dos pais relativas à preocupação com a frequência e a qualidade nas interações estabelecidas com filhos, no que diz respeito a brincadeiras e tarefas cotidianas. A relevância do fortalecimento da relação entre pais e filhos e sua influência no desenvolvimento psicossocial da criança foram assinalas pelos participantes deste estudo, corroborando os achados de Trahan (2018). Além disso, é fundamental ressaltar a oportunidade da participação paterna no Programa ACT, em alguns grupos de intervenção desta pesquisa. Os participantes homens puderam expor suas dúvidas, sendo reconhecidos e valorizados pelos demais na vivência da paternidade. Esse dado ratifica a importância da intervenção com a figura paterna (Benczik, 2011; Manfroi, 
Macarini, \& Vieira, 2011), uma vez que os estudos nacionais ainda estavam estritos à figura da mãe (Altafim et al., 2016).

Foi possível observar que os pais compreenderam a importância do estilo parental, a partir dos conteúdos trabalhados durante a intervenção. Os participantes conseguiram reconhecer que o seu próprio comportamento frente a diferentes situações acaba por tornarse referência para seus filhos. Esse resultado é bastante relevante, pois está de acordo com os princípios do Programa ACT, que concebem o aprendizado das crianças como um processo que depende do contexto social e ocorre por meio de observação, imitação de modelos e experiência direta (Knox et al., 2011; Silva, 2011; Silva \& Willians, 2016). Percebe-se, ainda, que os pais identificaram a relevância da adoção do estilo parental com autoridade, que pressupõe o estabelecimento de limites, porém sem emprego de autoritarismo e/ou de práticas punitivas/violentas, corroborando achados de investigações anteriores (Knox \& Burkhart, 2014; Pedro, 2016; Pontes, 2015; Silva \& Williams; 2016).

Outra importante constatação deste estudo é de que a participação dos pais no Programa ACT auxiliou no entendimento de que a formação do estilo parental é resultante de uma série de fatores, que abarcam os recursos culturais e socioeconômicos aos quais tiveram acesso, a relação com o outro genitor da criança, o estilo parental dos próprios pais, e as características específicas de cada filho (Belsky, 1984; Belsky \& Jaffee, 2006). Dessa forma, os conteúdos do programa e as discussões em grupo propiciaram aos participantes a oportunidade de perceber que, em alguns casos, não conseguiram fornecer um ambiente suficientemente satisfatório para o desenvolvimento saudável dos filhos devido a questões do histórico familiar em suas diferentes esferas. Verificou-se, portanto, um processo de desculpabilização por parte participantes do Programa ACT, à medida que compreenderam a parentalidade como uma tarefa bastante desafiadora (Pereira et al., 2015), o que provavelmente venha a colaborar para a ampliação do seu bem-estar psicossocial (Barlow, Smailagic, Huband, Roloff, \& Bennett, 2012) e consequentemente do bem- estar dos filhos (Sanders, 2008).

A inclusão da temática sobre as mídias eletrônicas é considerada um dos diferenciais do Programa ACT, se comparado a outros programas de intervenção que não abordam conteúdos sobre os meios eletrônicos (Altafim \& Linhares, 2016; Knox et al., 2011). Os apontamentos dos participantes explicitaram a tomada de consciência no que se refere ao cuidado com os conteúdos acessados nas mídias eletrônicas pelos filhos, bem como ao controle do tempo de exposição às mídias eletrônicas. Esse resultado está em conformidade com pesquisas quantitativas, que revelaram aumento do conhecimento a respeito dos efeitos das mídias eletrônicas no comportamento e no desenvolvimento infantil (Altafim et al., 2016; Camargo, 2016; Pedro, 2016; Porter \& Howe, 2008; Weymouth \& Howe, 2011). Conclui-se, dessa forma, que a inclusão da temática da mídia eletrônica no Programa ACT tem demonstrado efeitos positivos na percepção dos participantes.

No que concerne à compreensão sobre o desenvolvimento da criança, os pais demonstraram interesse em entender melhor o desenvolvimento infantil em suas diferentes fases, conforme apontado em estudos anteriores (Knox \& Burkhart, 2014; Weymouth \& Howe, 2011). Nesse sentido, destaca-se que o olhar mais atento às particularidades do desenvolvimento infantil por parte dos pais constitui um recurso emocional e cognitivo importante, que influencia diretamente no vínculo entre pais e filhos, bem como no comportamento infantil, contribuindo para que a criança estabeleça relações gratificantes ao longo da vida (Zuzarte \& Calheiros, 2010). Desse modo, os pais tiveram a oportunidade de aprender que determinados comportamentos considerados difíceis (como birra, por exemplo) são esperados no desenvolvimento infantil típico, dado que as crianças estão em 
processo contínuo de aprendizagem de comportamentos, compreendendo gradativamente como as pessoas e o mundo funcionam (Burkhart, Knox, \& Brockmyer, 2013; Knox \& Burkhart, 2014).

É valido destacar que este estudo contemplou pais e mães, sendo que alguns grupos foram mistos (homens e mulheres), mas verificou-se que a maioria dos concluintes foram mães. Este dado corrobora com trabalhos realizados com o Programa ACT a nível internacional que também aponta para a maciça participação feminina no programa (Knox et al., 2011; Knox et al., 2010; Weymouth \& Howe, 2011). Apesar dos estudos apontarem para a maior participação paterna na criação dos filhos, as mães permanecem assumindo boa parte da responsabilidade no cuidado e educação dos filhos, além de exercerem o trabalho remunerado (Dessen \& Ribeiro, 2013). Desta forma, são necessários investimentos em relação às funções das figuras parentais na educação infantil e os impactos da participação de ambos os pais para o pleno desenvolvimento infantil, sendo o programa de intervenção um dos espaços de interação e aprendizagem (Milkie, Bowling, \& Denny, 2015).

\section{Conclusões}

O presente estudo propôs-se a identificar as aprendizagens de participantes de grupos de pais do Programa ACT, intervenção psicoeducativa destinada a pais e cuidadores de crianças pequenas, cujo propósito é a prevenção universal de maus tratos contra a criança. As análises indicaram que a participação no Programa ACT auxiliou na aprendizagem: i)de práticas educativas parentais positivas; ii) de estratégias de disciplina positiva; iii) no reconhecimento de práticas educativas negativas; iv) na compreensão dos efeitos da exposição à violência na vida das crianças; v) na gestão emocional dos pais e dos filhos; vi) na compreensão acerca dos estilos parentais; vii) na importância do envolvimento dos pais no processo de desenvolvimento psicossocial e emocional das crianças; viii) no aumento do conhecimento sobre desenvolvimento infantil; e ix) na importância do controle da utilização de mídias eletrônicas pelas crianças.

Diante disso, destaca-se a relevância de intervenções psicoeducativas com pais e cuidadores de crianças, sobretudo programas parentais que ampliem o conhecimento acerca do desenvolvimento infantil, ensinem estratégias positivas de manejo com as crianças e incentivem relacionamentos familiares estáveis, seguros e harmoniosos. Este estudo deve o mérito de mostrar que os participantes do Programa ACT percebem os aspectos mais importantes das intervenções e compreendem sua relevância. Ademais, apresentou como contribuição às pesquisas do Programa ACT uma análise qualitativa de aspectos específicos concernentes às aprendizagens realizadas nas sessões e mostrou resultados de grupos mistos (pais e mães) no contexto brasileiro, diferenciando-se de estudos anteriores apenas com mães.

Quanto às limitações deste estudo, destaca-se a fato de terem sido analisadas apenas as anotações dos participantes no término das sessões do programa, assim como a pequena participação paterna nos grupos. Ressalta-se que a participação do pai em programas parentais ainda se configura como um importante desafio a ser superado. Sugere-se a realização de estudos futuros, longitudinais, que realizem entrevistas e grupos focais logo após a conclusão do programa e, também, por meio de follow up, para verificar se as aprendizagens referidas nos registros serão mantidas ao longo do tempo. Novos estudos do Programa ACT poderiam ser realizados com grupos mistos ou apenas com pais (homens) 
com o intuito de incentivar a participação paterna e promover o envolvimento do pai no desenvolvimento cognitivo, emocional e comportamental dos filhos.

Considera-se que pesquisas sobre intervenções parentais podem subsidiar políticas públicas que apoiem as famílias na desafiadora tarefa que é a parentalidade, devido aos inúmeros aspectos que a influenciam. Ademais, podem embasar a prática profissional de psicólogo(a)s, educadores e assistentes sociais que trabalham com famílias com crianças pequenas.

\section{Referências}

Altafim, E. R. P. (2017). Avaliação da eficácia de um programa de intervenção preventiva em práticas educativas parentais. Tese de doutorado. Universidade de São Paulo - USP.

Altafim, E. R. P., \& Linhares, M. B. M. (2016). Universal violence and child maltreatment prevention programs for parents: A systematic review. Psychosocial Intervention, 25(1), 27-38. doi: 10.1016/j.psi.2015.10.003

Altafim, E. R. P., McCoy, D. C., \& Linhares, M. B. M. (2018). Relationships between parenting practices, socioeconomic status, and child behavior in Brazil. Children and Youth Services Review, 89, 93-102. doi: 10.1016/j.childyouth.2018.04.025

Altafim, E. R. P., Pedro, M. E., \& Linhares, M. B. M. (2016). Effectiveness of ACT Raising Safe Kids Parenting Program in a developing country. Children and Youth Services Review, 70, 315-323. doi: 10.1016/j.childyouth.2016.09.038

Bandura, A. (1977). Social learning theory. England Cliffs, NJ: Prentice-Hall.

Barbeta, M., \& Cano, T. (2017). Toward a new model of fatherhood? Discourses on paternal involvement in urban Spain. Revista Española de Investigaciones Sociológicas, 159, 13-30. doi: 10.5477/cis/reis.159.13

Bardin, L. (2011). Análise de conteúdo: edição revisada e ampliada. São Paulo: Edições 70.

Barlow, J., Smailagic, N., Huband, N., Roloff, V., \& Bennett, C. (2012). Group-based parent training programs for improving parental psychosocial health. Cochrane Database of Systematic Reviews, 13(6), CD002020. doi: 10.1002/14651858.CD002020.pub3

Belsky, J. (1984). The determinants of parenting: A process model. Child Development, 55(1), 83-96. doi: 10.2307/1129836

Belsky, J, \& Jaffee, S. (2006). The multiple determinants of parenting. Em D. Cicchetti, \& D. Cohen (Eds). Developmental psychopathology (2da ed.) (pp. 38-77). Nova York: John Wiley.

Benczik, E. B. P. (2011). A importância da figura paterna para o desenvolvimento infantil. Revista Psicopedagogia, 28(85), 67-75.

Boden, M. T., \& Thompson, R. J. (2015). Facets of emotional awareness and associations with emotion regulation and depression. Emotion, 15(3), 399-410. doi: 10.1037/emo0000057

Burkhart, K. M., Knox, M., \& Brockmyer, J. (2013). Pilot evaluation of the ACT raising safe kids program on children's bullying behavior. Journal of Child and Family Studies, 22(7), 942-951. doi: 10.1007/s10826-012-9656-3

Camargo, A. P. L. (2016). Programa ACT de prevenção à violência: Implementação e evidências de efetividade em amostra carcerária feminina do Estado do Paraná (Dissertação demestrado não publicada). Universidade Tuiuti do Paraná. 
Conselho Nacional de Saúde Resolução $n^{\circ}$ 466/2016. Disponível em: http://conselho.saude.gov.br/resolucoes/2012/reso466.pdf. Acesso em: 30 de novembro de 2020.

Dessen, M. A., \& Polonia, A. C. (2007). A família e a escola como contextos de desenvolvimento humano. Paidéia, 17(36), 21-32. doi: 10.1590/S0103863X2007000100003

Dessen, M. A. \& Ribeiro, O. M. (2013). Envolvimento Paterno Durante o Nascimento dos Filhos: Pai "Real" e "Ideal" na Perspectiva Materna. Psicologia: Reflexão e Crítica, 26(1), 184-192. doi: 10.1590/S0102-79722013000100020

Gulliford, H., Deans, J., Frydenberg, \& Liang, R. (2015). Teaching coping skills in the context of positive parenting within a preschool setting. Australian Psychologist, 50(3), 219-231. doi: 10.1111/ap.12121

Knox, M., \& Burkhart, K. (2014). A multi-site study of the ACT Raising Safe Kids program: predictors of outcomes and attrition. Children and Youth Services Review, 39, 20-24. doi: 10.1016/j.childyouth.2014.01.006

Knox, M., Burkhart, K., \& Cromly, A. (2013). Supporting positive parenting in community health centers: The ACT Raising Safe Kids Program. Journal of Community Psychology, 41(4), 395-407. doi: 10.1002/jcop.21543

Knox, M., Burkhart, K., \& Howe, T. (2011). Effects of the ACT Raising Safe Kids parenting program on children's externalizing problems. Family Relations, 60(4), 491-503. doi: 10.1111/j.1741-3729.2011.00662.x

Knox, M., Burkhart, K., \& Hunter, K. E. (2010). ACT Against Violence Parents Raising Safe Kids Program: Effects on maltreatment-related parenting behaviors and beliefs. Journal of Family Issues, 32(1), 55-74. doi: 10.1177/0192513X10370112

Lamb, M. E., Pleck, J. H., Charnov, E. L., \& Levine, J. A. (1985). Paternal behavior in humans. American Zoologist, 25, 883-894.

Linhares, M. B. M. (2015). Família e desenvolvimento na primeira infância: Processos de autorregulação, resiliência e socialização de crianças pequenas. In G. A. Pluciennik, M. C. Lazzari, \& M. F. Chicaro (Orgs.), Fundamentos da família como promotora do desenvolvimento infantil: Parentalidade em foco (pp. 70-82). São Paulo, SP: Fundação Maria Cecília Souto Vidigal.

Manfroi, E.; Macarini, S.M., \& Vieira, M. L. (2011). Comportamento parental e o papel do pai no desenvolvimento infantil. Revista Brasileira de Crescimento e Desenvolvimento Humano, 21(1), 59-69. doi: 10.7322/jhgd.19996

Miguel, J. J., \& Howe, T. R. (2006). Implementing \& evaluating a national early violence prevention program at the local level: Lessons from ACT (Adults \& Children Together) Against Violence. Journal of Early Childhood \& Infant Psychology, 2, 17-38.

Milkie, M. A., Bowling, K. M. N. \& Denny, K. E. (2015). Does the Amount of Time Mothers Spend with Children or Adolescents Matter? Journal of Marriage and Family, 77, 355372. doi: 10.1111/jomf.12170

Pedro, M. E. (2016). Programa ACT - para educar crianças em ambientes seguros para promover práticas educativas maternas positivas em diferentes contextos socioeconômicos. Dissertação de mestrado. Universidade de São Paulo - USP.

Pereira, A. I. F., Goes, A. R., \& Barros, L. (2015). Promoção da parentalidade positiva: intervenções psicológicas com pais de crianças e adolescentes. Lisboa: Coisas de Ler.

Pontes, L. B. (2015). Avaliação da eficácia de programa de capacitação parental universal (ACT) pelo uso da observação. Dissertação de Mestrado: Universidade Federal de São Carlos - UFSCar. 
Porter, B., \& Howe, T. (2008). Pilot evaluation of the "ACT Parents Raising Safe Kids" violence prevention program. Journal of Child \& Adolescent Trauma, 1(3), 193-206. doi: 10.1080/19361520802279158

Portwood, S. G., Lambert, R. G., Abrams, L. P., \& Nelson, E. B. (2011). An evaluation of the Adults and Children Together (ACT) Against Violence Parents Raising Safe Kidsprogram. Journal of Primary Prevention, 32(3-4), 147-160. doi: 10.1007/s10935011-0249-5

Sanders, M. R. (2008). Triple P-Positive Parenting Program as a public health approach to strengthening parenting. Journal of Family Psychology, 22(4), 506-517. doi: 10.1037/0893-3200.22.3.506

Sanders, M. R., Kirby, J. N., Tellegen, C. L., \& Day, J. J. (2014). The Triple P-Positive Parenting Program: A systematic review and meta-analysis of a multi-level system of parenting support. Clinical Psychology Review, 34, 337-357. doi: 10.1016/j.cpr.2014.04.003

Schmidt, B., Gomes, L. B., Bossardi, C. N., Bolze, S. D. A., Vieira, M. L. \& Crepaldi, M. A. (2019). Envolvimento parental e temperamento de crianças: uma revisão sistemática da literatura. Contextos clínicos, 12(1), 75-103. doi: 10.4013/ctc.2019.121.04

Silva, J. (2011). Programa ACT para Educar Crianças em Ambientes Seguros, Manual do Facilitador e Guia de Avaliação. Washington, DC: American Psychological Association.

Silva, J. A., \& Williams, L. C. A. (2016). Um estudo de caso com o Programa Parental ACT para educar crianças em ambientes seguros. Temas em Psicologia, 24(2), 743-755. doi: 10.9788/TP2016.2-19Pt

Trahan, M. H. (2018). Paternal Self-Efficacy and Father Involvement: A Bi-Directional Relationship. Psychology of Men \& Masculinity, 19(4), 624-634. doi: 10.1037/men0000130

Webster-Stratton, C., Reid, M. J., \& Hammond, M. (2001). Preventing conduct problems, promoting social competence: A parent and teacher training partnership in Head Start. Journal of Clinical Child Psychology, 30(3), 283-302. doi: 10.1207/S15374424JCCP3003_2

Weymouth, L. A., \& Howe, T. (2011). A multi-site evaluation of Parents Raising Safe Kids Violence Prevention Program. Children and Youth Services Review, 33(10), 1960-1967. doi: 10.1016/j.childyouth.2011.05.022

Zuzarte, M., \& Calheiros, M. (2010). Programa de intervenção nas interacções pais-filhos "Desenvolver a Sorrir" - Estudo exploratório. Análise Psicológica, 3(28), 491-504. doi: 10.14417/ap. 350

Participação dos autores: Participação dos autores: a) Planejamento e concepção do trabalho; b) Coleta de dados; c)Análise e interpretação de dados; d) Redação do manuscrito; e) Revisão crítica do manuscrito.

J. L. A. P. contribuiu em a, b, c, d, e; M. C. F. em a, b, c, d, e; L. G. em a, b, c, d, e; M. L. V. em a, e; M. A. C. em a, e.

Editora científica responsável: Dra. Cecilia Cracco 